\title{
Endoskopische Resektionsverfahren EMR und ESD - Schritt für Schritt
}

Andreas Probst, Manuel Pagitz, Helmut Messmann, Jörg Albert

\begin{abstract}
Prämaligne oder frühe maligne Läsionen des Gastrointestinaltrakts können endoskopisch entfernt werden - und diese organerhaltende Therapie bietet eine erheblich bessere Lebensqualität für die Patienten als eine Organresektion. Verschiedene Verfahren stehen zur Verfügung: Grundsätzlich unterschieden werden die endoskopische Mukosaresektion (EMR) und die endoskopische Submukosadissektion (ESD), die in diesem Beitrag detailliert dargestellt werden.
\end{abstract}

\begin{tabular}{|c|c|}
\hline \multicolumn{2}{|l|}{ ABKÜRZUNGEN } \\
\hline CSI-EMR & $\begin{array}{l}\text { zirkumferenzielle submuköse } \\
\text { Inzision und EMR }\end{array}$ \\
\hline EMR & $\begin{array}{l}\text { endoskopische Mukosa- } \\
\text { resektion }\end{array}$ \\
\hline EMR-cap, EMR-C & $\begin{array}{l}\text { Kappen-Mukosektomie- } \\
\text { Technik }\end{array}$ \\
\hline EMR-ligation, EMR-L & $\begin{array}{l}\text { EMR in Kombination mit } \\
\text { Gummibandligatur }\end{array}$ \\
\hline ER & endoskopische Resektion \\
\hline ESD & $\begin{array}{l}\text { endoskopische Submukosa- } \\
\text { dissektion }\end{array}$ \\
\hline LST & Laterally spreading Tumor \\
\hline NBI & Narrow Band Imaging \\
\hline pEMR & Piecemeal-Resektion \\
\hline
\end{tabular}

\section{Einführung}

\section{Endoskopische Mukosaresektion}

Die EMR kommt bei flächig wachsenden Neoplasien zum Einsatz. Bei allen Varianten der EMR wird ein Flüssigkeitsdepot in die Submukosa unterhalb einer abzutragenden Läsion gespritzt ( $\triangleright$ Abb. 1). Hierdurch wird die Läsion angehoben („Lifting“), der Abstand zwischen Resektionslinie und Muskulatur vergrößert und damit die Perforationsgefahr im Rahmen der Resektion reduziert.

Für die Resektion selbst stehen dann verschiedene Varianten zur Verfügung. Bei der konventionellen EMR wird die Läsion nach der Unterspritzung mit der Schlinge gefasst und dann mit Hochfrequenzstrom abgetragen. Bei der Kappen-Mukosektomie-Technik (EMR-cap, EMR-C) wird eine Aufsatzkappe auf das Endoskop platziert und in der Kappe eine geöffnete Schlinge platziert. Die abzutragende Läsion wird nun in die Kappe eingesaugt, durch
Zuzug der Schlinge gefasst und dann mit Hochfrequenzstrom reseziert. Eine Alternative zur EMR-C ist die EMR in Kombination mit Gummibandligatur (EMR-ligation, EMR-L). Hierbei wird das abzutragende Schleimhautareal in die aufgesetzte Kappe eingesaugt, mit einer Gummibandligatur abgebunden und danach das „ligierte“ Gewebe mit einer Elektroschlinge reseziert.

Als Nachteil der EMR gilt die Limitation des Resektionsausmaßes durch die verwendeten Schlingen bzw. Kappen. Bei größeren Läsionen bzw. immer bei Läsionen mit einem Durchmesser von > 20 mm muss die EMR als Resektion in mehreren Stücken erfolgen (Piecemeal-Resektion, pEMR). Neben dem Risiko der inkompletten Resektion steigt dabei auch das Rezidivrisiko. Nahezu immer handelt es sich dann bei Rezidiven um wieder gewachsene Reste der initial durch die pEMR nicht komplett entfernten Läsion.

\section{Endoskopische Submukosadissektion}

Die in Japan entwickelte Technik der endoskopischen Submukosadissektion (ESD) erlaubt eine Resektion auch großer Läsionen in einem Stück (en bloc).

\footnotetext{
Merke

Vorteile der ESD sind eine verbesserte histopathologische Beurteilung hinsichtlich einer R0-Situation sowie ein nahezu komplett eliminiertes Rezidivrisiko.
}

Nachteile der ESD sind der höhere Zeit- und Materialaufwand, eine flache Lernkurve und ein erhöhtes Perforationsrisiko. Im Gegensatz zur EMR erfolgt die Resektion nicht mit Schlingen, sondern mit speziellen Messern und anderen Instrumenten. 


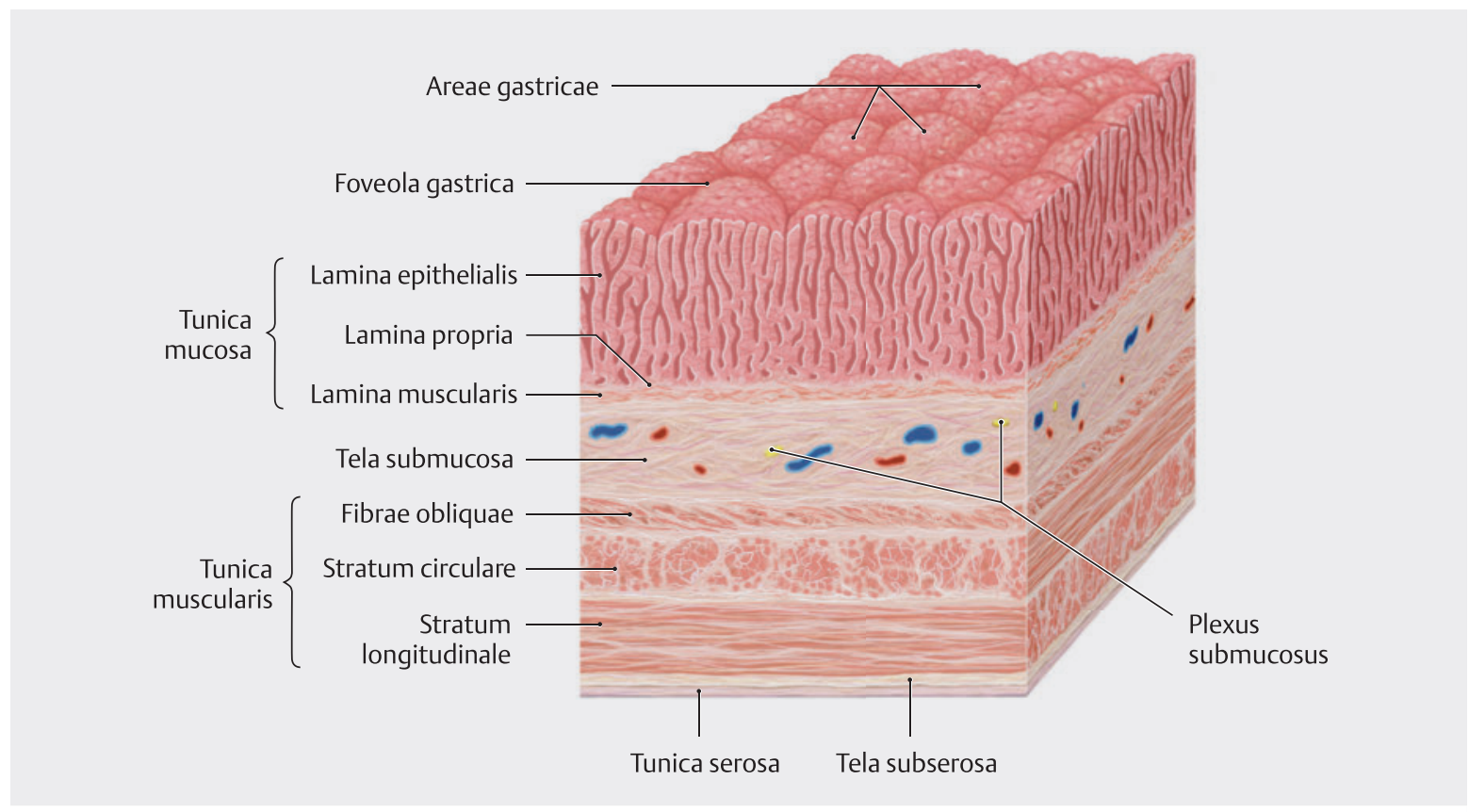

- Abb. 1 Aufbau der Schleimhaut des Verdauungstraktes am Beispiel des Magens. Die oberflächlichen Schichten umfassen die Mukosa und die Submukosa. Tief liegt die Tunica muscularis. (Quelle: Schünke M, Schulte E, Schumacher U et al., Hrsg. Prometheus LernAtlas - Innere Organe. Illustrationen von M. Voll und K. Wesker. 4. Auflage. Thieme; 2015. doi:10.1055/b-004-129727).

\section{Auswahl des Resektionsverfahrens}

Initial muss eine endoskopisch detektierte Läsion endoskopisch hinsichtlich ihrer Dignität und - im Falle eines Frühkarzinoms - ihrer mutmaßlichen Infiltrationstiefe eingeschätzt werden. Die Beurteilbarkeit des Malignitätsrisikos ist entscheidend, um abzuschätzen, ob eine Enbloc-Resektion notwendig ist, und entsprechend das optimale Resektionsverfahren zu wählen (EMR vs. ESD). Bestandteile der endoskopischen Diagnostik sind die Beurteilung der Wachstumsform (Paris-Klassifikation) sowie die anschließende Feinbeurteilung des Oberflächenreliefs (Surface Pattern) und der Gefäßarchitektur (Vascular Pattern). Am Ende der makroskopischen Beurteilung sollte eine Einschätzung der technischen Resektabilität sowie der onkologisch sinnvollen Resektabilität (kurative Resektion) stehen.

\section{Merke \\ Bei frühmalignen Befunden (vorliegende Histologie oder Verdacht aufgrund morphologischer Kriterien) sollte eine En-bloc-Resektion der Läsion unbedingt angestrebt werden.}

Sollte eine En-bloc-Resektion malignitätsverdächtiger Läsionen mittels EMR nicht sicher möglich sein, sollte der ESD der Vorzug gegeben werden. Bei makroskopisch benigne eingestuften Befunden müssen die Vor- und Nachteile der EMR und ESD im Einzelfall abgewogen werden.

\section{Indikationen für die ESD}

Derzeit in Europa akzeptierte Indikationen für eine Resektion in ESD-Technik sind

- alle Plattenepithelfrühkarzinome des Ösophagus, wenn kein Verdacht auf eine Submukosainvasion besteht,

- ausgewählte Adenofrühkarzinome des Ösophagus (Durchmesser von mehr als $15 \mathrm{~mm}$, schlechtes Lifting nach Unterspritzung oder Verdacht auf Submukosainvasion),

- Adenome und Frühkarzinome des Magens, die einer endoskopischen Resektion (ER) aus onkologischer Sicht zugänglich sind. Sie sollten immer mittels ESD abgetragen werden.

Im Kolorektum sollte eine ESD immer dann erwogen werden, wenn die Morphologie eines Polypen auf ein mögliches Malignitätsrisiko hinweist:

- Laterally spreading Tumors (LST) vom Granular Type mit größeren Knoten

- LST vom Nongranular Type

- eingesenkte Läsionen

- Läsionen mit malignomverdächtiger Oberflächenoder Gefäßarchitektur [1].

Für benigne kolorektale Läsionen stellt die EMR heute noch das Standardverfahren dar. Im Einzelfall sollte aber auch hier der Vorteil eines nahezu eliminierten Rezidivrisikos nach ESD individuell in die Wahl des Resektions- 
verfahrens einbezogen werden. Dies gilt insbesondere im Rektum, wo die ESD im Vergleich zum Kolon ein sehr geringes Komplikationsrisiko aufweist [2].

\section{Alternativen zu EMR und ESD}

Eine weitere endoskopische Abtragungsmöglichkeit stellt die Kombination der beiden Techniken EMR und ESD dar: CSI-EMR (circumferential submucosal incision endoscopic mucosal resection). Dabei wird z.B. der LST in ESD-Technik zirkumferenziell umfahren und somit die Mukosa inzidiert; anschließend wird der Polyp mit der Schlinge in EMR-Technik abgetragen. Damit wird an den lateralen Rändern eine vollständige Entfernung gewährleistet und so die Rezidivrate im Vergleich zur klassischen EMR gesenkt - die Perforationsrate ist dabei vergleichbar niedrig wie bei der klassischen EMR. Bei mittelgroßen Polypen $(2-4 \mathrm{~cm})$ kann es durch das zirkumferenzielle Umfahren in einigen Fällen zu einem „Schrumpfen“ des Polypen kommen. Dann kann dieser im Anschluss doch en bloc mittels Schlinge reseziert werden. Die postulierten Vorteile (niedrige Perforationsrate bei niedrigem Rezidivrisiko) dieser kombinierten Abtragungstechnik müssen jedoch noch in prospektiven Studien bestätigt werden.

Läsionen unter 20 mm können in Fällen eines „Non-Liftings“ mit der endoskopischen Vollwandresektion behandelt werden, da hier eine transmurale Resektion möglich ist [3].

\section{Merke}

Eine operative Resektion von benignen Adenomen ist heute nur sehr seltenen Ausnahmefällen vorbehalten, jedoch als alternative Therapieoption grundsätzlich möglich.

Unter gewissen Bedingungen ist jedoch eine primäre Operation die Therapie der Wahl, z.B.

- bei familiärer adenomatöser Polyposis (FAP),

- bei hochgradigen Dysplasien im Rahmen einer Colitis ulcerosa oder

- wenn die fragliche Läsion nach klinischen und endoskopischen Parametern verdächtig auf eine fortgeschrittene Invasionstiefe ist oder ein Lymphknotenbefall zu vermuten ist $[4,5]$.

\section{Schritt 1 Vorbereitung des Patienten}

Notwendig ist eine Aufklärung über den Eingriff einschließlich Sedierung (Hinweis auf z. B. Nachblutung, Perforation, aber auch inkomplette Resektion mit notwendiger sekundärer Operation etc.). Im Falle der Koloskopie ist eine vollständige Darmreinigung nötig. Voraussetzung für eine ER sind zudem eine ausreichende Blutgerinnung (INR < 1,5; PTT < 50 s; Thrombozyten > $50 \mathrm{G} / \mathrm{I})$.

\section{Schritt 2}

\section{Vorbereitung der Endoskopie}

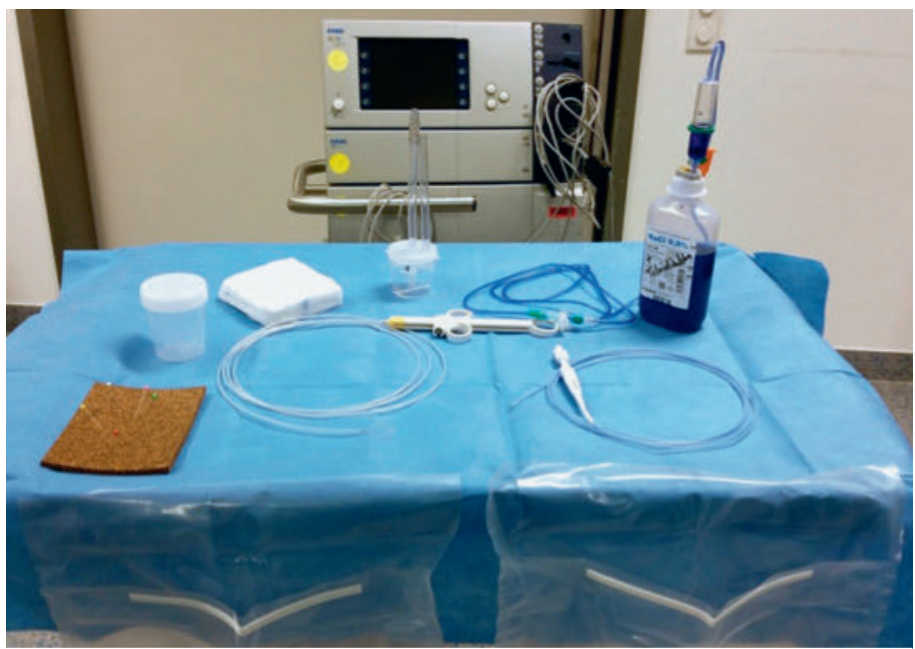

- Abb. 2 Vorbereiteter Tisch mit Korkplatte, Formalin, Schlinge, Nadel zur Unterspritzung, Kompressen, Polypenfalle und physiologischer Kochsalzlösung mit Toluidinblau.

Zusätzlich zur regulären Vorbereitung der Endoskopie (z. B. Geräte-Check, Monitoring) empfiehlt es sich, eine Färbelösung (z.B. Toluidinblau $300 \mathrm{mg}$ ad $500 \mathrm{ml} \mathrm{NaCl}$ $0,9 \%$ ) für die submuköse Injektion vorzubereiten. Es folgt die Vorbereitung der Elektrochirurgie (einschließlich korrektem Anbringen einer Nullelektrode). Des Weiteren werden Probengläser (Formaldehyd 4\%) oder ein Korkblock zum Aufspannen des Resektats benötigt ( $\bullet$ Abb. 2).

Die Auswahl des passenden Endoskops hängt von der Lage des Polypen ab: Im Rektum kann ein flexibles therapeutisches Gastroskop Vorteile bieten, im Colon ascendens ein klassisches Koloskop. Es empfiehlt sich, hochauflösende Videogeräte der letzten Generation einzusetzen, wir verwenden auch immer eine kurze, weiche Aufsatzkappe.

Aktuell erfolgt die ESD in unseren Kliniken unter stationären Bedingungen. Resektionen im Ösophagus und im proximalen Magen führen wir in Intubationsnarkose durch. Ansonsten erhält der Patient eine tiefe intravenöse Sedierung mit Midazolam und Propofol durch einen hierfür qualifizierten Arzt. Eine Antibiotikaprophylaxe erfolgt nicht routinemäßig. 


\section{Schritt 3}

\section{Detektion und Charakterisierung}

Mit hochauflösender Videoendoskopie wird die Läsion bei optimaler Vorreinigung des Ösophagus, Magens oder Darms sicher erkannt. Optische Filterverfahren des Lichtes oder eine konventionelle Chromoendoskopie helfen bei der Einschätzung, ob beispielsweise ein hyperplastischer oder adenomatöser Polyp vorliegt. Klassifikationen (s. „Info - Klassifikation“) ermöglichen eine Risikoabschätzung der Entartungswahrscheinlichkeit oder submukösen Invasivität einer u.U. bereits malignen Läsion im oberen Gastrointestinaltrakt ( $\mathbf{A}$ Ab. $\mathbf{3}$ ) oder Kolon ( Abb. 4).
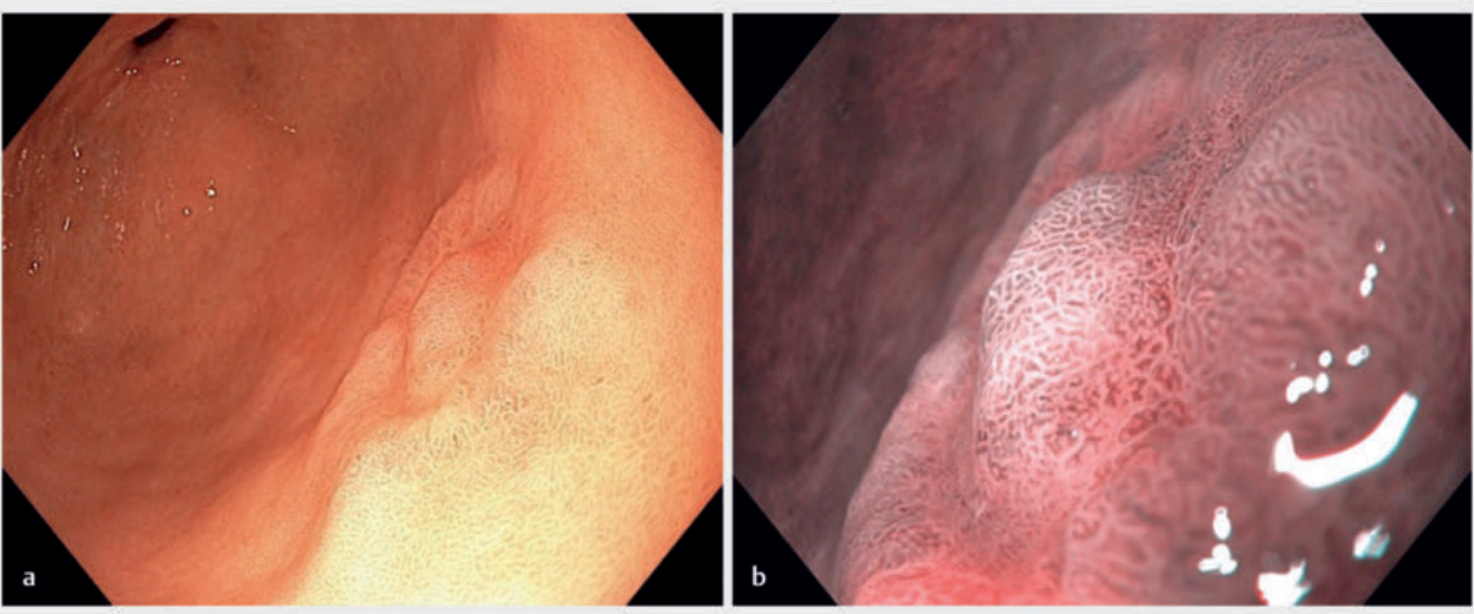

Abb. 3 Endoskopische Diagnostik einer Magenfrühneoplasie (Biopsie: Adenom mit leichter Dysplasie - Morphologie: dringender Verdacht auf Magenfrühkarzinom).

a Weißlichtendoskopie.

b Narrow Band Imaging (NBI).
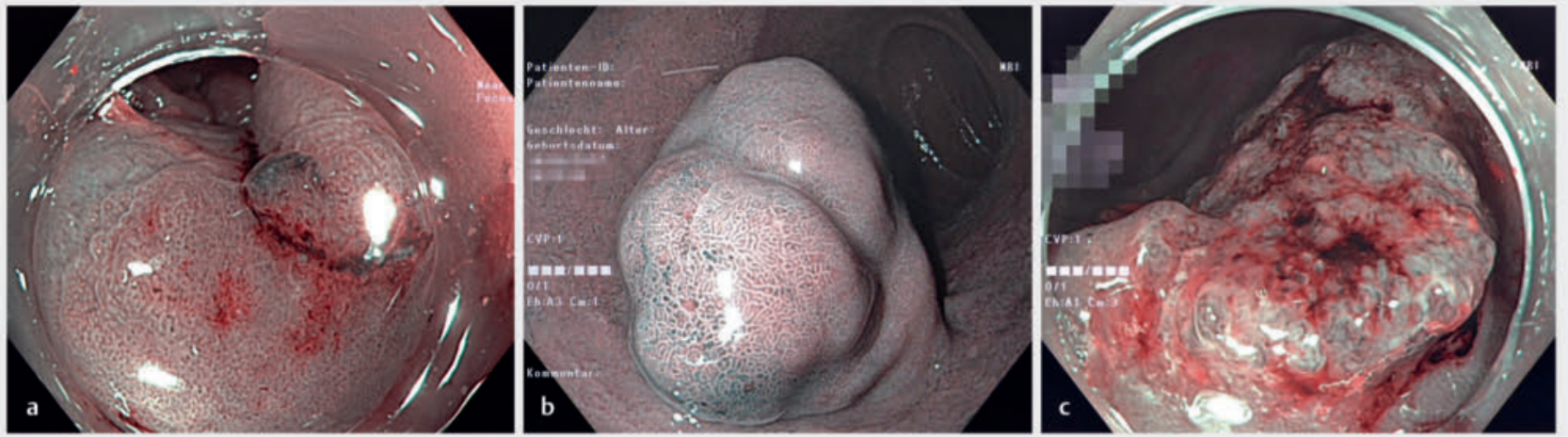

Abb. 4 Charakterisierung von kolorektalen Polypen.

a Flache Läsion im Colon ascendens: NICE 1/WASP-positiv (Oberfläche glatt, Begrenzung scharf, Form unregelmäßig, vereinzelt Krypten mit dunklen Punkten: somit 2 positive WASP-Kriterien) als Hinweis auf ein sessil serratiertes Adenom.

b Adenom NICE 2.

c Areale von unterbrochenen oder fehlenden Gefäßen und amorphes bzw. fehlendes Oberflächenmuster: NICE 3, bereits Verdacht auf submukosal infiltrierendes Karzinom. 


\section{INFO}

\section{Klassifikation}

Folgende Klassifikationssysteme dienen der Charakterisierung von frühen gastrointestinalen Neoplasien:

- Paris-Klassifikation: Morphologie des Polypen (• Abb. 5)

- Kudo-Klassifikation: „pit pattern“

- Sano-Klassifikation: „capillary pattern“

- NICE-Klassifikation „Narrow Band Imaging International Colorectal Endoscopic Classification“: Beispiel für die Anwendung von optischen Filterverfahren für die Charakterisierung von kolorektalen Polypen, validiert für Polypen $<1 \mathrm{~cm}$ $(\triangleright$ Tab. 1)

- WASP-Klassifikation „Workgroup on serrAted polypS and Polyposis Classification“: ergänzt NICE-Kriterien um Hazewinkel-Kriterien zur Abgrenzung serratierter Adenome von hyperplastischen Polypen. Bei NICE-Typ-1- oder -2-Läsionen werden folgende Kriterien abgeprüft (sind 2 davon erfüllt, handelt es sich am ehesten um ein sessil serratiertes Adenom):

- wolkenartige Oberfläche

- unscharfer Rand

- dunkle, winzige Grübchen/Pünktchen in den Krypten

- unregelmäßige Form
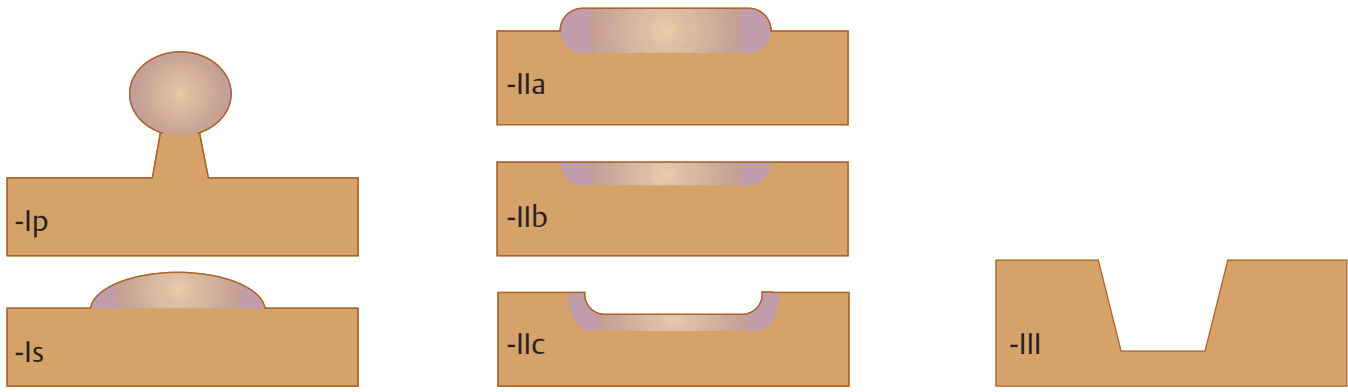

- Abb. 5 Paris-Klassifikation zur morphologischen Einteilung oberflächlicher gastrointestinaler Neoplasien. Typ I: vorgewölbt (gestielt Ip, sessil Is), Typ II: flach (flach-erhaben Ila, flach-flach Ilb, flach-eingesenkt Ilc), Typ III: ulzeriert (Quelle: Probst A. Polypen und Polyposis. In: Messmann H, Hrsg. Lehratlas der Koloskopie. 2., aktualisierte Aufl. Thieme; 2014. doi:10.1055/b-002-96283).

- Tab. 1 NICE-Klassifikation.

\begin{tabular}{|l|l|l|l|l|}
\hline Typ & $\begin{array}{l}\text { Farbe (im Vergleich } \\
\text { zur Umgebung) }\end{array}$ & Gefäße/Gefäßmuster & $\begin{array}{l}\text { Oberflächenstruktur } \\
\text { Histologie }\end{array}$ & hyperplastischer Polyp \\
\hline Typ 1 & $\begin{array}{l}\text { gleich oder heller als } \\
\text { der Hintergrund }\end{array}$ & $\begin{array}{l}\text { a: keine Gefäße } \\
\text { b: filigran geschwungen } \\
\text { c: kleine Pünktchen umgeben } \\
\text { von weißen Kreisen }\end{array}$ & $\begin{array}{l}\text { dunkle oder weiße Pünktchen } \\
\text { in homogener Anordnung }\end{array}$ & \\
\hline Typ 2 & $\begin{array}{l}\text { braun, dunkler (beachte } \\
\text { gefäßbedingte Färbung) }\end{array}$ & $\begin{array}{l}\text { braun, homogen, von weißen } \\
\text { Strukturen umgeben }\end{array}$ & $\begin{array}{l}\text { ovale, tubuläre, verzweigte } \\
\text { Strukturen, umgeben von } \\
\text { braunen Gefäßen }\end{array}$ & Adenom \\
\hline Typ 3 & $\begin{array}{l}\text { dunkelbraun, fleckige } \\
\text { weiße Areale }\end{array}$ & $\begin{array}{l}\text { irregulär, unterbrochen, } \\
\text { teils fehlend }\end{array}$ & $\begin{array}{l}\text { amorph, destruiertes oder } \\
\text { fehlendes Relief }\end{array}$ & Karzinom \\
\hline
\end{tabular}




\section{Schritt 4}

\section{Resektionsgrenzen und -strategie}

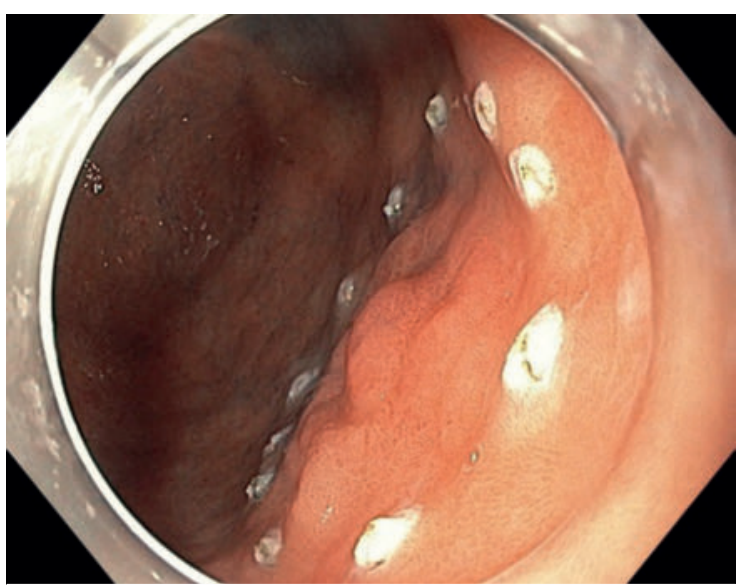

- Abb. 6 Markierung der Resektionsgrenzen durch Koagulationsmarken außerhalb der Läsion.

Nach abgeschlossener morphologischer Diagnostik müssen die Resektionsgrenzen und die Therapiestrategie der ER festgelegt werden. Insbesondere im Ösophagus und Magen kann die Bestimmung der lateralen Ausdehnung einer Läsion schwierig und manchmal erst durch Chromoendoskopie, Vergrößerungsendoskopie und z.B. NBI möglich sein.

\footnotetext{
Merke

Im Ösophagus und Magen müssen die Resektionsgrenzen vor Beginn der Abtragung unbedingt durch Koagulationsmarken gekennzeichnet werden.
}

Dies kann bei der ESD am einfachsten mit der Spitze des für die Resektion vorgesehenen Messers erfolgen ( $\bullet$ Abb. 6). Alternativen sind eine APC-Sonde (ArgonPlasma-Koagulation) oder die Spitze der Resektionsschlinge. Im Kolorektum ist eine Markierung nur bei sehr flachen Läsionen erforderlich; polypoide Läsionen sind hier auch nach einer Unterspritzung noch gut nach lateral abgrenzbar.

Im Vergleich zu anderen Resektionstechniken spielt die Lage einer Läsion im Verhältnis zur Schwerkraft bei der ESD eine besondere Rolle. Die Lagerung des Patienten sollte bereits initial so erfolgen, dass die Schwerkraft (erkennbar an der Lage intraluminaler Flüssigkeit) der abzutragenden Läsion möglichst gegenüberliegt.

\section{Schritt 5 Unterspritzung}

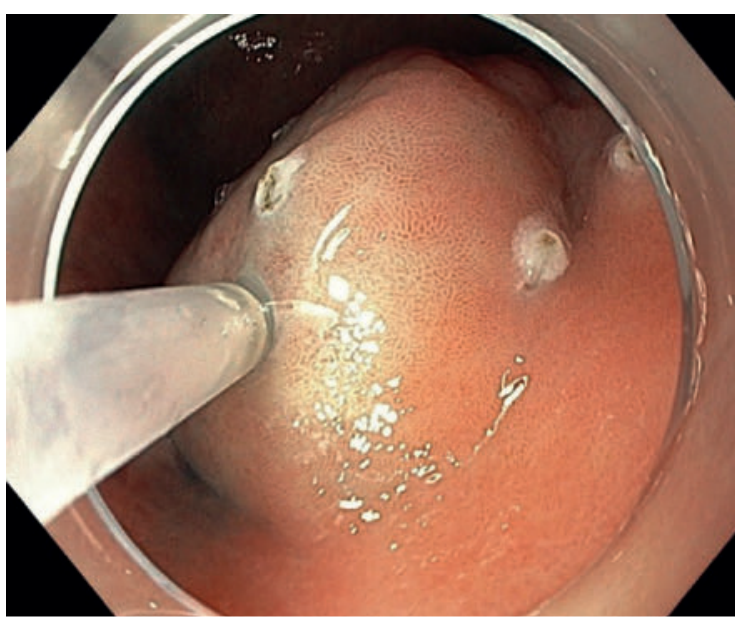

Abb. 7 Unterspritzung der Läsion außerhalb der markierten Resektionsgrenzen.

Spätestens jetzt sollte eine transparente Abstandskappe auf das Distalende des Endoskops aufgesetzt werden. Die Unterspritzung erfolgt bei der ESD analog zur EMR ( $\bullet$ Abb. 7).

\section{Merke}

Das Ziel der Unterspritzung ist, den Durchmesser der Submukosa zu vergrößern (Lifting) und so das Risiko einer Verletzung der Muscularis propria bzw. das Perforationsrisiko zu minimieren.

Zur Injektion kann prinzipiell jede Substanz verwendet werden; die Auswahl ist untersucherabhängig. Für die EMR verwenden wir Kochsalzlösung mit Adrenalin (Konzentration Adrenalin 1:100000). Zur ESD bevorzugen wir eine 10\%ige Glycerollösung mit Adrenalin (Endkonzentration 1:50000-1:100000) und einer geringen Menge Indigokarmin. Bei schlechtem Lifting kann der Einsatz von Hyaluronsäure sehr hilfreich sein; nachteilig sind die hier höheren Kosten.

Ab diesem Punkt unterscheidet sich das Vorgehen für eine ESD von einer EMR und entsprechend werden die weiteren Schritte getrennt betrachtet! 


\section{Schritt 6 EMR: Resektion}

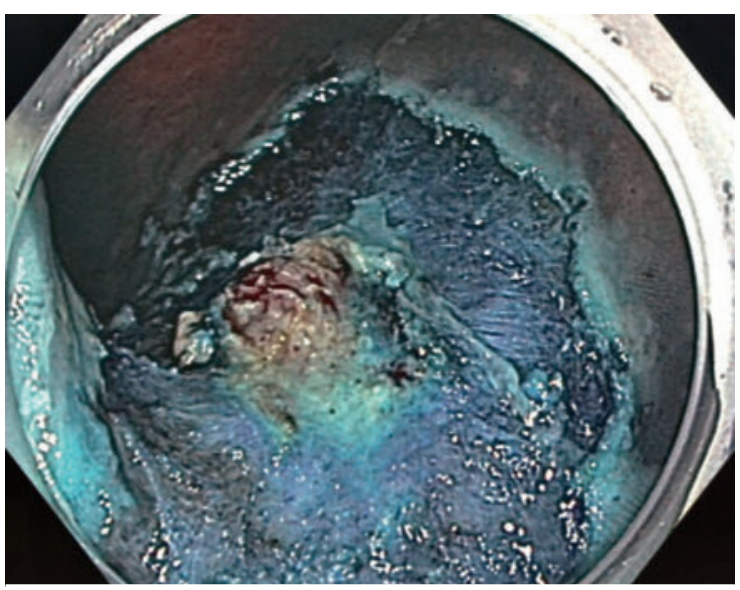

- Abb. 8 Komplett abgetragene Läsion mit Sicht auf den Resektionsgrund.

Voraussetzung für eine optimale Resektion ist die geeignete Lage des Endoskops ohne Schleifenbildung (Kontrolle des Schaftes, Übertragung von Drehbewegungen direkt auf die Spitze). Die Ausrichtung des Polyps sollte unterhalb der Austrittsstelle des Arbeitskanals im Bild sein (auf $6 \mathrm{Uhr}$ ). Das gewählte Instrument (Schlinge, Kappe oder Gummiband) wird nun ausgebracht und platziert.

Bei Resektion mit der Schlinge wird diese leicht von der Wand zum Zentrum des Lumens hin bewegt, damit ein möglichst geringer thermischer Effekt auf die Wand einwirkt. Nach elektrochirurgischer Abtragung der Läsion werden die Resektionsränder zum Ausschluss von Resten der Läsion intensiv inspiziert ( $\mathbf{A} \mathbf{A b} \mathbf{b} . \mathbf{8})$.

\section{Schritt 6 ESD: Mukosa- durchtrennung, Umschneidung}

Nach der Unterspritzung wird die Mukosa außerhalb der markierten Resektionsgrenzen durchtrennt. Hierzu stehen verschiedene Messer zur Verfügung.

Merke

Die Durchtrennung der Mukosa sollte die Submukosa eröffnen und in ihrer Tiefe nahe an die Muscularis propria reichen, ohne diese jedoch zu berühren.

Eine zu flache Inzision erschwert nicht nur die Erreichbarkeit der Submukosa, sondern erhöht auch das Blutungsrisiko durch inkomplette Koagulation submukosaler Gefäße.

Ob die Umschneidung initial komplett um die gesamte Läsion (zirkumferenziell) oder zunächst nur partiell erfolgt, hängt von Größe, Lage und Erreichbarkeit der abzutragenden Läsion ab. Eine zunächst nur partielle Inzision hat den Vorteil einer besseren Traktion des noch nicht abgetragenen Gewebes während der submukosalen Dissektion. Zu beachten ist aber, dass Bereiche einer noch nicht komplett erfolgten Inzision im Verlauf der Abtragung eventuell schwierig erreichbar werden können (Beispiel: aboraler Rand einer Ösophagusläsion, wenn die oralseitig partiell abgetragene Läsion nach aboral umklappt). Die Komplettierung einer initial nur partiellen Umschneidung muss im weiteren Verlauf der ESD rechtzeitig erfolgen. Die beste Abtragungsstrategie sollte dementsprechend bereits zu Beginn der Resektion festgelegt werden ( Abb. 9).

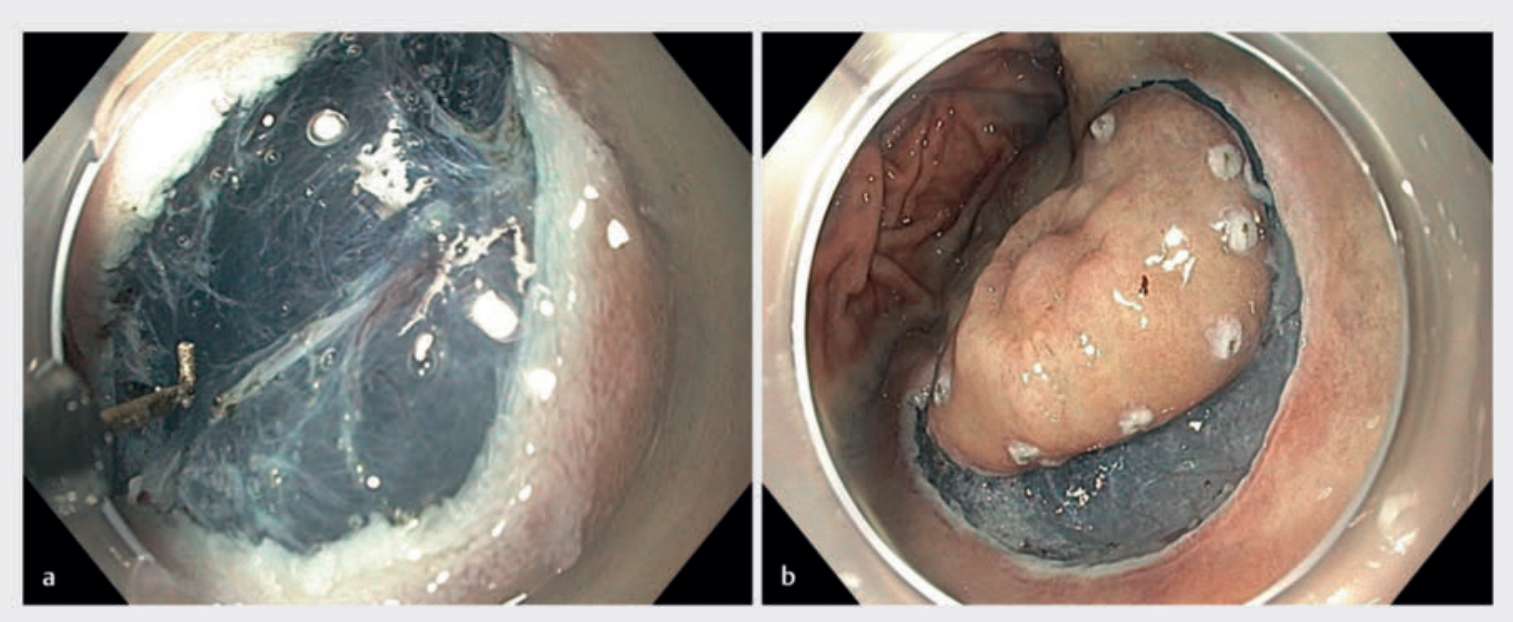

Abb. 9 Mukosadurchtrennung und Umschneidung.

a Inzision der Mukosa, hier mittels Hook Knife (KD-620LR, Olympus).

b Komplette zirkumferenzielle Umschneidung der Läsion. 


\section{Schritt 7 ESD: Submukosadissektion}

Nach erfolgter Inzision wird die Submukosa mit dem hierzu ausgewählten Messer durchtrennt. Eine wiederholte Unterspritzung in die Submukosa ist während der Submukosadissektion erforderlich ( $\triangleright$ Abb. 10). Als Alternative zur konventionellen Unterspritzung mittels Injektionsnadel stehen mittlerweile verschiedene Messer mit integriertem Injektionskanal zur Verfügung. Diese erlauben neben der Schneide- und Koagulationsoption auch eine Unterspritzung. Vorteil ist der fehlende Zeitverlust durch den ansonsten erforderlichen Instrumentenwechsel. Die Auswahl des Messers ist untersucherabhängig.

Innerhalb der Submukosa vorhandene Gefäße können entweder prophylaktisch vor ihrer Durchtrennung oder spätestens bei eingetretener Blutung koaguliert werden. Die Koagulation kleiner Gefäße kann mit der Spitze des ESD-Messers erfolgen; für größere Gefäße kann der Einsatz zusätzlicher Koagulationszangen erforderlich sein.

\section{Schritt 8 ESD: Nach komplettierter Abtragung}

Nach Komplettierung der Resektion wird das Resektat geborgen und das Abtragungsulkus inspiziert. Erkennbare größere Blutgefäße sollten zur Prophylaxe einer verzögerten Blutung mit der Spitze des ESD-Messers oder einer Koagulationszange koaguliert werden. Das Resektat wird mit Stecknadeln auf eine Kork- oder Gummiplatte aufgespannt und zur histopathologischen Untersuchung versandt ( $\triangleright$ Abb. 11).

\section{Nach der Intervention}

\section{ESD: Beachtenswertes}

Nach Resektionen im oberen Gastrointestinaltrakt empfehlen wir eine Therapie mit Protonenpumpenhemmern: zunächst in doppelter Standarddosis für 6 Wochen und dann in einfacher Standarddosis für weitere 6 Wochen bis zur ersten endoskopischen Kontrolle.

Merke

Zu beachten ist das Risiko einer postinterventionellen Strikturentwicklung bei Resektionen im Ösophagus, wenn die Resektion mehr als 75\% der Zirkumferenz umfasst.

Hier ist eine Strikturprophylaxe obligat (lokale oder systemische Steroidtherapie, prophylaktische endoskopische Dilatation). Derartige Resektionen sollten ausschließlich in hierfür erfahrenen Zentren erfolgen.

\section{EMR: Nachsorge}

Bei größeren Läsionen mit höherem Komplikationsrisiko kann eine stationäre Überwachung indiziert sein. Die Empfehlung zur Kontrollkoloskopie richtet sich nach der Vollständigkeit der Abtragung sowie dem histologischen Ergebnis. Bei Piecemeal-Technik oder gar klinisch inkompletter Resektion zum Eingriffsende wird eine Kontrollkoloskopie nach 4-6 Monaten empfohlen.
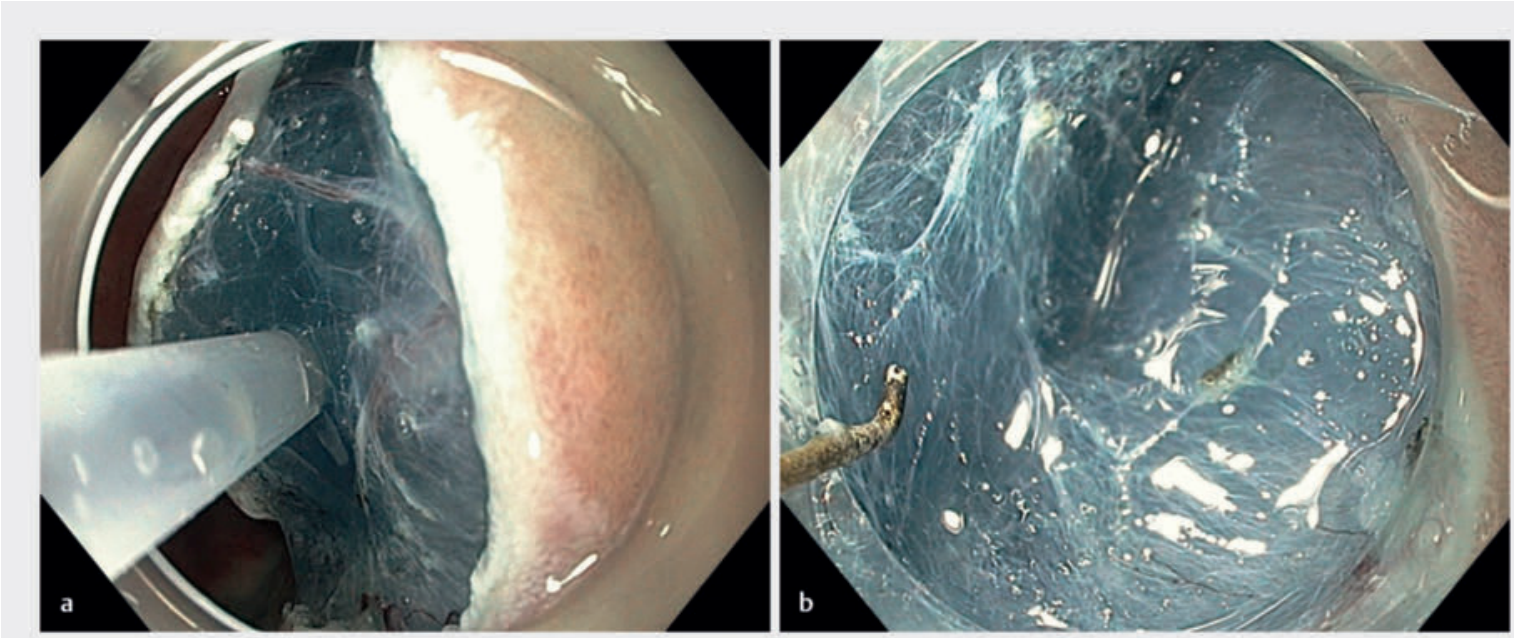

Abb. 10 Submukosadissektion.

a Erneute Unterspritzung während der Dissektion.

b Dissektion der Submukosa, hier mittels Hook Knife (KD-620LR; Olympus). 

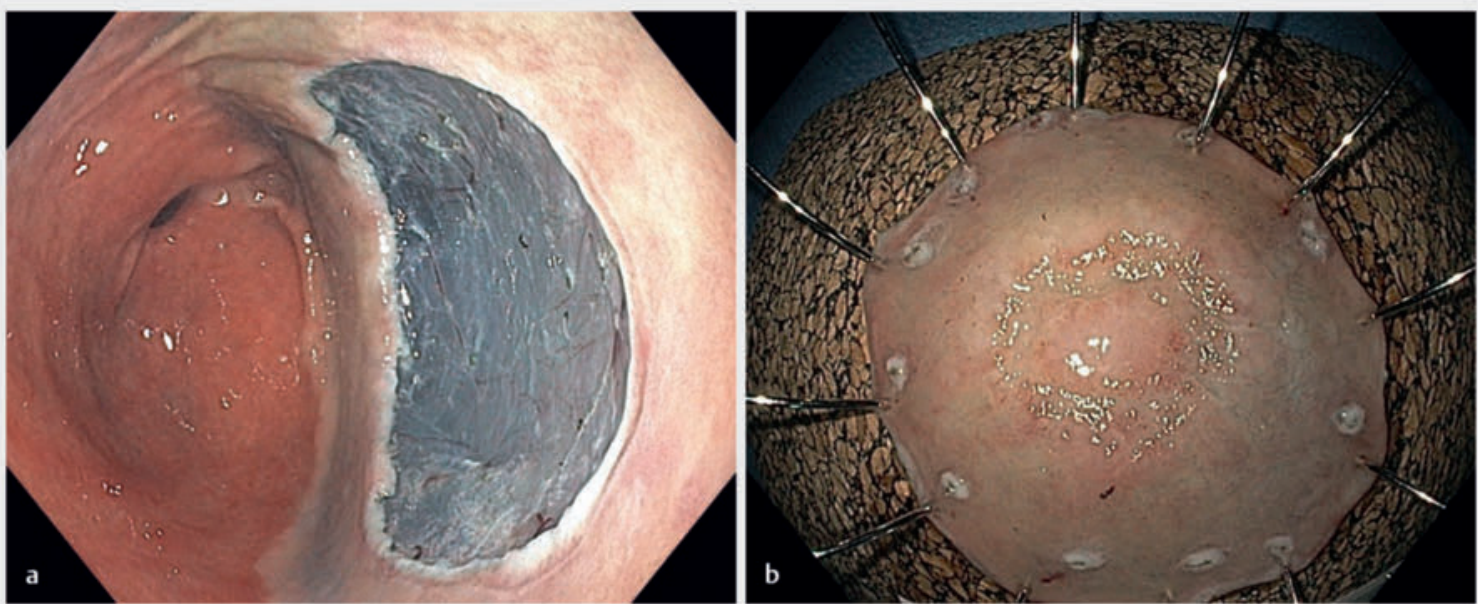

- Abb. 11 Nach der ESD.

a Resektionsulkus nach komplettierter ESD.

b Resektat auf Korkplatte aufgespannt (Histologie: Magenfrühkarzinom pT1a L0 V0 G1 R0).

\section{Zusammenfassung}

EMR und ESD sind minimalinvasive Resektionsverfahren, mit denen frühe Neoplasien im Gastrointestinaltrakt schnell und sicher entfernt werden können. Sorgfältig beachtet werden müssen die Kriterien wie Größe der Läsion, Erhabenheit und Eingesenktheit. Die ER ist bei Dysplasien und Frühkarzinomen hinsichtlich Lebensqualität und Kosten der radikalen Operation deutlich überlegen.

\section{Interessenkonflikt}

J. Albert: Vortragshonorare Olympus, Medtronic, FujiFilm, Falk. Die weiteren Autoren geben an, dass keine Interessenkonflikte vorliegen.

\section{Autorinnen/Autoren}

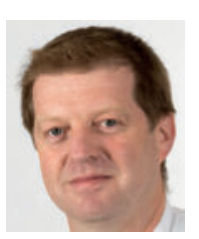

\section{Andreas Probst}

Dr. med., 1988-1994 Studium der Humanmedizin an der LMU München. 2001 Facharzt für Innere Medizin, 2002 Teilgebietsbezeichnung Gastroenterologie, 2009 Zusatzbezeichnung Medikamentöse Tumortherapie. Seit 2015 Leitender Oberarzt an der III. Medizinischen Klinik des Klinikums Augsburg. Klinische Schwerpunkte: Therapeutische Endoskopie, Gastroenterologische Onkologie.

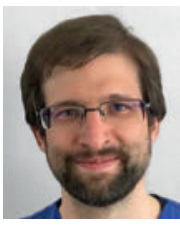

\section{Manuel Pagitz}

Geboren 1984 in Nagold, Studium der Humanmedizin in Frankfurt am Main. Bis 2016 Facharztausbildung Innere Medizin am Klinikum Frankfurt Höchst, aktuell in Weiterbildung zum Gastroenterologen am Robert-Bosch-Krankenhaus Stuttgart.

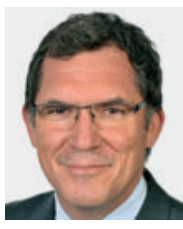

\section{Helmut Messmann}

Prof. Dr. med., Chefarzt der III. Medizinischen Klinik des Klinikums Augsburg. Forschungsschwerpunkte: Endoskopische Früherkennung und Therapie von Präkanzerosen und Frühkarzinomen im Gastrointestinaltrakt, Interventionelle Endoskopie, Entwicklung moderner Technologien für die diagnostische und therapeutische Endoskopie, Gastroenterologische Onkologie, Internistische Intensivmedizin.

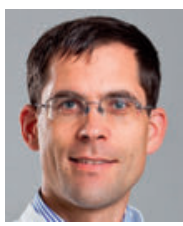

\section{Jörg Albert}

Prof. Dr. med., Facharzt für Innere Medizin und Gastroenterologie. Chefarzt am Robert-BoschKrankenhaus, Stuttgart. Leiter der Abteilung für Gastroenterologie, Hepatologie und Endokrinologie. Klinische Schwerpunkte: Interventionelle Viszeralmedizin, Gastroenterologische Onkologie. Mitglied zahlreicher Fachgesellschaften, u. a. der Deutschen Gesellschaft für Endoskopie und bildgebende Verfahren (DGE-BV) und der European Society of Gastrointestinal Endoscopy (ESGE). 
Prof. Dr. med. Jörg Albert

Robert-Bosch-Krankenhaus

Abteilung für Gastroenterologie, Hepatologie

und Endokrinologie

Auerbachstraße 110

70376 Stuttgart

joerg.albert@rbk.de

Erstveröffentlichung

Dieser Beitrag ist eine aktualisierte Version des Artikels: Pagitz M, Koch M, Hausmann J, Albert J. Endoskopische Mukosaresektion (EMR) - Schritt für Schritt. Dtsch Med Wochenschr 2018; 143: 1032-1038.
[1] Pimentel-Nunes P, Dinis-Ribeiro M, Ponchon T et al. Endoscopic Submucosal Dissection: European Society of Gastrointestinal Endoscopy (ESGE) Guideline. Endoscopy 2015; 47: 829854

[2] Probst A, Ebigbo A, Märkl B et al. Endoscopic submucosal dissection for early rectal neoplasia: experience from a European center. Endoscopy 2017; 49: 222-232

[3] Rosenberg N. Submucosal saline wheal as safety factor in fulguration or rectal and sigmoidal polyp. AMA Arch Surg 1955; 70: 120-122

[4] Ferlitsch M, Moss A, Hassan C et al. Colorectal polypectomy and endoscopic mucosal resection (EMR): European Society of Gastrointestinal Endoscopy (ESGE) Clinical Guideline. Endoscopy 2017; 49: 270-297. doi:10.1055/s-0043-102569

[5] Kamiński MF, Hassan C, Bisschops R et al. Advanced imaging for detection and differentiation of colorectal neoplasia: European Society of Gastrointestinal Endoscopy (ESGE) Guideline. Endoscopy 2014; 46: 435-449. doi:10.1055/s-0034-1365348

Bibliografie

DOI https://doi.org/10.1055/s-0044-100308 Gastroenterologie up2date 2018; 14: 309-319 (c) Georg Thieme Verlag KG Stuttgart · New York ISSN 1616-9670

\section{Schon} registriert?

Bei Thieme CME Punkte online einreichen und verwalten

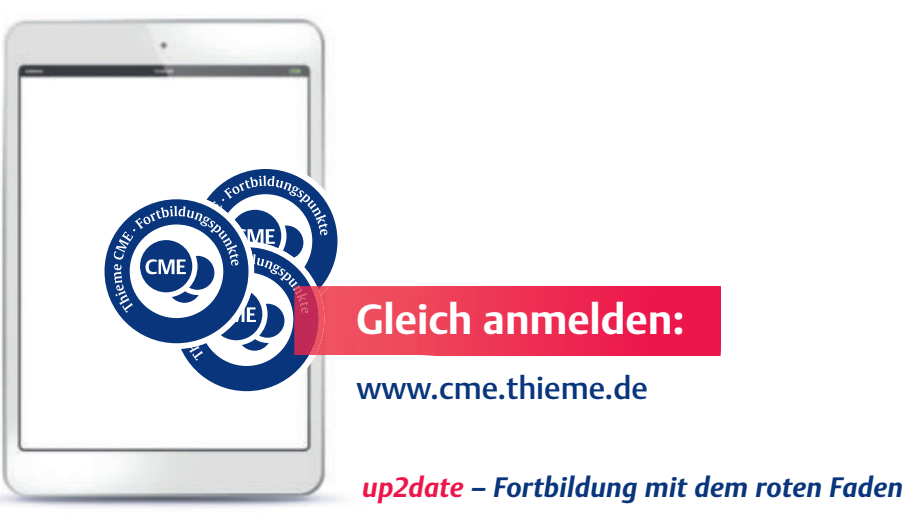

